\title{
CONCERNANT LES DIVERSES CLASSIFICATIONS \\ PROPOSÉES POUR LES DERMATOPHYTES
}

\author{
Par R. SABOURAUD
}

Depuis Bazin, on réserve le nom ancien de teignes aux affections épidermiques déterminées par des champignons.

Depuis la découverte du premier de ces parasites, l'Achorion, par Schönleïn (1839) et depuis la découverte de trois autres parasites analogues par Grúby (1842-1845), le nombre des affections cutanées déterminées par des champignons inférieurs n'a pas cessé de s'accroître. Ce qu'on croyait d'abord causé par un, ou deux, ou trois parasites distincts, s'est trouvé causé par une cinquantaine..., d'où la nécessité d'une classification.

Or, dans leur vie parasitaire, sur l'homme et les animaux, ces moisissures ne se présentent que sous deux formes, toutes deux

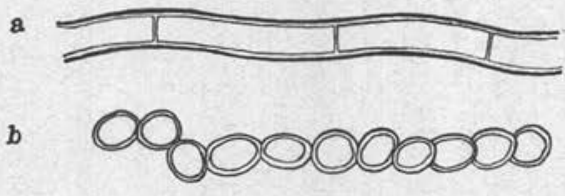

Fı́ 1. - Articles mycéliens dans les squames épid rmiques.

mycéliennes. Tantòt leurs filaments ressemblent à des tiges de bambou, dont chaque nœud marque une cloison intercellulaire. C'est la forme que tous ces parasites prennent dans l'épiderme corné de surface (fig. 1, a). Tantôt ces filaments ressemblent à un chapelet. Les articles mycéliens sont devenus plus gros, plus courts, un peu globuleux et leur écorce cellulosique s'est épaissie (fig. 1, b). C'est la forme ordinaire que ces parasites prennent dans le cheveu ou autour de lui. Bien que chacun de ces articles soit apte à reproduire le parasite, c'est plutôt comme une bouture que comme une graine. Néanmoins, la langue dermatologique appelle "spores " (1) ces articles courts, par une impropriété de terme certaine. Ce sont les premiers naturalistes saisis de la question par Bazin qui

(1) Je crois que ces soi-disant spores doivent être assimilées aux arthrospores des cultures glabres des dermatophytes dont il sera question plus loin.

AnNales de Parasttologie, $\mathrm{T}$. VII, $\mathrm{N}^{\circ} 3 .-1^{\text {er }}$ mai 1929 , p. 228-246. 
crurent voir, en ces chaînes d'articles, des spores vraies, émises bout à bout, comme il se voit en certaines espèces cryptogamiques, et le nom leur resta.

Done, les parasites des teignes ne montrent jamais, sur le vivant, aucun organe vrai de reproduction. Ceci est le premier point à retenir.

Et maintenant, disons la forme objective que les lésions de

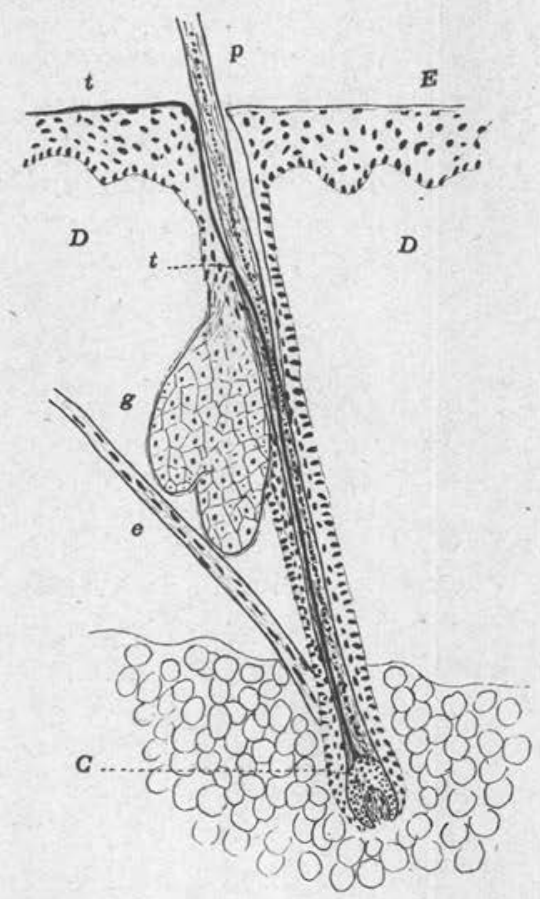

Fig. 2. - Mode d'envahissement des poils par les dermatophytes. $E$, épiderme ; $D$, derme; $p$, poil ou cheveu; $g$, glande sébacée; $e$, muscle érecteur du poil ; $C$, collet du bulbe du poil ; $t$, trait noir indiquant le chemin suivi par le parasite.

toutes les teignes affectent communément à leur début. Comme la semence portée en un point émet autour d'elle des rayons filamenteux égaux, la lésion produite est orbiculaire. Toute lésion d'une teigne quelconque peut affecter cette forme ronde; et comme le plus souvent son centre meurt, la peau y redevient saine, tandis que la circonférence reste rose, vésiculeuse ou desquamante sur un demi-centimètre de large ; c'est ce que les dermatologistes anciens appelaient l'herpès circinè (érusıv, serpere, ramper). 
Normalement ces parasites ne trouvent à vivre que dans l'épiderme corné, " kératinisé ", mais le poil et le cheveu sont kératinisés mème dans la profondeur de la peau. Si done un filament parasitaire rencontre l'ouverture d'un follicule pileux, il s'incurve avec l'épiderme et descend avec lui dans le follicule ( $t$, fig. 2). Là, il còtoie le cheveu, tissu corné, il l'envahit de haut en bas, descendant vers sa base en se ramifiant au point de le remplir même en entier. Capables seulement de proliférer dans l'épiderme corné, ces parasites ne peuvent pas envahir le poil jusqu'au point où il prend naissance, car en ce point sa kératinisation n'est pas faite (elle se fait au point dit : collet du bulbe pilaire) (C, fig. 2). La fonction qui fait le cheveu ou le poil n'est donc nullement troublée par ce parasitisme. Le cheveu continue de pousser, mais à mesure qu'il pousse de bas en haut, il se kératinise ; alors le parasite poussant de haut

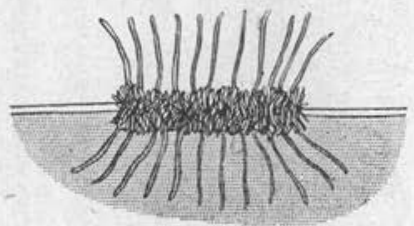

Fig. 3. - Schéma de la culture d'un dermatophyte.

en bas envahit la partie nouvellement cornée. Ce phénomène curieux fait la permanence in situ de teignes qui dureront des années et quelquefois autant que la vie du malade (favus).

Résumons ce qui précède :

La double forme de ces parasites : dans l'épiderme = filaments mycéliens ; et dans le cheveu = arthrospores en chapelet...

La forme orbiculaire commune au début de toutes leurs lésions épidermiques de surface...

L'envahissement du cheveu rendu permanent par le mode physiologique de la croissance du cheveu et de la croissance inverse du parasite... Telles sont les premières notions indispensables à connaître dans le sujet.

Gultures. - Cependant, ces parasites, transportés de leur lésion humaine sur des milieux de culture artificiels s'y développent pour la plupart comme des moisissures communes. Ils émettent dans la profondeur des filaments nourriciers comme des racines, au-dessus desquelles se développe un thalle épais et feutré. De sa surface sont 
émis des rameaux aériens dont beaucoup se couvrent d'organes différenciés de reproduction (fig. 3).

Or l'aspect objectif que prendra chacun de ces parasites en culture, sur des milieux appropriés, reste admirablement le même quand il s'agit dú même parasite, et différent quand il s'agit d'un parasite différent.

On a done désigné et nommé ces parasites d'après leur aspect spécifique sur le même milieu, et on en a décrit autant d'espèces que les cultures montraient de types fixes, immuables en leurs caractères et différents de tous autres (1).

Désormais la question des teignes avait done deux faces: une face dermatologique et une face mycologique.

Dermatologie. - Cliniquement, tous ces parasites ne donnent pas lieu à des lésions identiques. Une teigne faveuse, peu contagieuse, d'aspect croùteux, née au cours de l'âge scolaire, mais qui durera autant que la vie du patient, n'est pas la même chose qu'une teigne tondante, bien plus contagieuse, qui ne montre pas de croûte et qui ne vivra que chez l'enfant, guérissant toute seule aux alentours de la puberté...

Il y a donc des types cliniques, ayant chacun leurs symptômes, leurs mœurs, leurs caractères différentiels, leur évolution et leur traitement propre.

Pour le clinicien, un favus n'est pas une teigne tondante et voir confondre ces deux maladies lui produit le même effet qu'à l'oreille d'un lettré un barbarisme.

Cependant le dermatologiste n'est pas un mycologue. Il a pu étudier ses cultures de teignes, découvrir et signaler leurs organes de reproduction, les décrire et les figurer, il n'en reste pas moins incapable de savoir dans quelle famille de mucédinées leurs organes les classent, d'apprécier leurs affinités familiales et de dire leur place parmi les autres.

Mycologie. - D'autre part, un mycologue ne verra jamais que des cultures qu'il a reçues des mains d'un dermatologiste. Les questions cliniques ne l'intéressent pas. Il s'occupera de placer ses cultures dans un ordre pour lui rationnel, sans s'inquiéter du type et des mœurs de la lésion d'où ils furent extraits, ef non pas même du type morphologique que ces parasites peuvent prendre dans le cheveu. Ce que j'en dis est pour faire toucher du doigt la difficulté

(1) Un des meilleurs milieux d'épreuve est fait de 1 p. 100 de peptone, 8 p. 100 de miel d'aheille, 100 d'eau et de la gélose, Q. S. pour solidifier. 
du problème. Il faudrait, pour le résoudre, un dermatologiste qui fût mycologue, ou inversement; cela ne s'est jamais rencontré.

Une extrême confusion s'en est suivie. Pour les mycologues, les nomenclatures dermatologiques n'ont pas de valeur botanique, et quand les mycologues présentent aux dermatologistes une classification qui leur parait plus rationnelle, les dermatologistes n'en veulent pas. Pour les dermatologistes, les mycologues édifient des classifications de cabinet qui ne correspondent à aucune réalité clinique vérifiable.

Pourtant, on ne modifie pas à son gré des faits d'expérience. Telle maladie a son type objectif, microscopique, évolutif, différentiel de tout autre. Telle culture de parasite cutané montre tels organes de reproduction. Ce sont là des faits naturels certains et qui ont assurément leur raison d'être ce qu'ils sont.

Il faudrait donc que chaque dermatologiste essayât de comprendre les arguments des mycologues et inversement. Le présent travail n'a pas d'autre but que de tendre vers cette alliance et de la préparer.

Principes directeurs. - Quelques principes pratiques et vérifiables peuvent nous $\mathbf{y}$ aider beaucoup.

$1^{\circ}$. - Le premier est la personnalité que toute culture neuve de dermatophyte prend sur certains milieux convenus, préparés suivant les techniques nécessaires.

Au Musée de Parasitologie de l'Hôpital St-Louis, n'importe qui, sur le seul aspect. de la culture, nommera de suite et sans crainte d'erreur, non seulement le Microsporum audouini, l'Achorion du favus banal ou le Trichophyton crateriforme, qui affectent trois types de culture de tous points différents, mais on différenciera au premier coup d'œil deux variétés très voisines : Microsporum lanosum et felineum, par exemple.

On a donc, dans des conditions de travail suffisamment attentives, un moyen de séparation et d'identification des espèces parasitaires ou variétés fixes.

$2^{\circ}$. - Le second principe est le suivant :

Sur un milieu sucré, presque toutes les cultures de dermatophytes s'altèrent assez vite, par l'apparition à leur surface de duvets blanes, irréversibles au type cultural originel. Tandis que sur milieu peptonisé sans sucre, les cultures réensemencées tous les deux mois gardent, on peut dire indéfiniment, leur propriété et leur type, aptes à le reprendre au premier ensemencement sur milieu sucré d'épreuve. Ainsi gardera-t-on ses souches-mères sans altẻration. 
$3^{\circ}$. - Et voici le troisième principe :

La figure extérieure d'une culture 'n'est que la traduction pour notre oeil des organes qui la constituent et que le microscope nous y montrera.

a. Ainsi le champignon d'une culture glabre ne présente que des filaments mycéliens " en bambous " ou " en chapelets ", très analogues à ceux du même être dans sa vie parasitaire. Ce sont des articles mycéliens ronds ou cubiques ou polyédriques, avec des facettes d'articulation quand ils sont tassés, ou libres et arrondis (fig. 4).

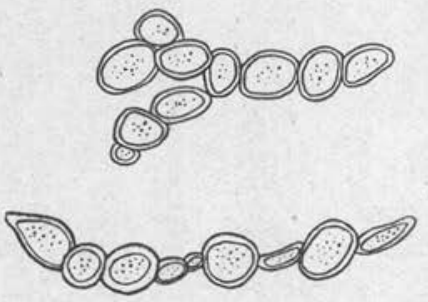

Fic. 4. - Arthrospores des cultures glabres.

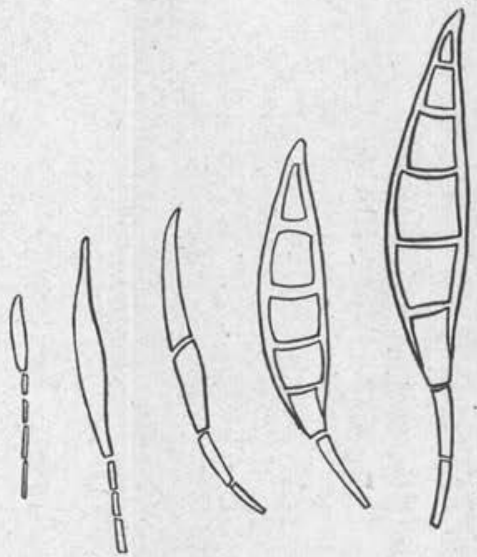

Fic. 5. - Petits fuseaux des cultures finement duveteuses et gros fuseaux des cultures duveteuses opaques.

$b$. Une culture finement duveteuse pour notre œil nous montrera de fins rameaux aériens sans organes différenciés ou quelquefois ces rameaux sont terminés par un renflement en forme de fuseau étroit unicellulaire.

c. Une culture duveteuse vivace qui devient lentement teintée et opaque montrera, au long des rameaux aériens ou à leur extrémité, des fuseaux pluriseptés plus ou moins longs, gros et nombreux (fig. 5).

$d$. Une culture poudreuse et d'aspect cartonneux montrera des filaments aériens courts, supportant, pédiculées ou sessiles, de petites cellules oblongues que j'avais appelées, sur l'avis de Duclaux: "spores externes ou conidies 》 et que les mycologues désirent appeler " aleuries" maintenant. Peu importent les noms si l'on s'entend bien sur les choses... 
Et tantòt ces aleuries seront espacées sur un long rameau mycélien, comme les fleurs d'une hampe de digitale ; tantôt elles seront agminées en grappes semblables à des grappes de raisin (fig. 6).

$e$. Cette disposition en grappes énormes est plus frappante encore dans les cultures plàtreuses, qui nous offrent aussi des aleuries en thyrses, des fuseaux moyens et quelquefois des vrilles ou spirales très semblables aux vrilles de la vigne (fig. 7).

Ainsi donc : des cultures glabres ne montrant que des éléments mycéliens difformes et des réserves protoplasmiques enkystées.
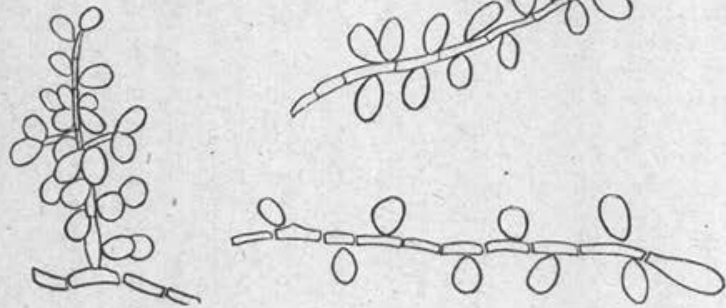

FIG, 6. - Aleuries disposées en thyrses et en grappes.

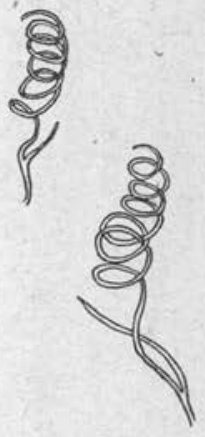

Fig. 7. - Spirales de quelquescultures plâtreuses.

Les duveteuses, des filaments stériles portant des aleuries plus ou moins rares et des fuseaux plus ou moins petits.

Les cartonneuses, des aleuries à foison.

Les duveteuses qui deviennent opaques : aleuries rares, mais gros fuseaux en quantité.

Les duveteuses qui deviennent plâtreuses : aleuries en thyrses et en grappes, fuseaux moyens, et, chez certaines, des vrilles ou spirales.

Bien entendu, une énumération si succincte laisse de côté les caractères botaniques individuels et reste dans les généralités.

Dès lors, si nous promenons nos regards sur toutes ces cultures comme sur des plantes dans un jardin, il nous semblera (puisque les caractères objectifs des cultures correspondent très exactement aux caractères botaniques des organes de reproduction, lesquels servent à établir toutes les classifications botaniques), il semblera, dis-je, que nous devrions mettre en un même tas toutes les cultures glabres, assurés de ne placer côte à còte que celles qui n'auraient pas d'organes de reproduction différenciés ; 
En un deuxième groupe, toutes les cultures finement duveteuses ;

En un autre, les cultures duveteuses qui deviennent opaques et ternes en vieillissant ;

En un autre, les cultures cartonneuses poudreuses ;

Et en un dernier, toutes les cultures duveteuses qui deviennent plâtreuses.

D'après ce que nous venons d'exposer tout à l'heure, nous serions certains d'avoir ainsi réuni, en un même groupe, les espèces dont les organes différenciés sont analogues. C'est ce que j'avais fait remarquer ici-même, lorsque je critiquais la classification nouvelle des dermatophytes apportée par MM. Ota et Langeron.

Ainsi, mème à l'œil nu, nous aurions fait un tri, correspondant à une classification d'apparence rationnelle, et l'examen microscopique des cultures semblerait en confirmer la véracité. C'est, à peu près, ce qu'ont fait tous les mycologues.

Ils ont rapproché dans un mème groupe tous les parasites dont les cultures étaient glabres; dans un autre, ceux dont les cultures étaient poudreuses, etc..., etc..., parce que si elles se ressemblent objectivement, elles se ressemblent mycologiquement.

Bien entendu, un tel résumé des opinions botaniques émises sur le sujet ne vise ni un homme, ni une classification, mais un ensemble d'opinions ; il les résume donc à grands traits. Pour être le plus court possible et impersonnel, il n'en reste pas moins véridique.

Garactères biologiques. - Maintenant, abandonnons le côté botanique de la question et envisageons la série des recherches biologiques faites sur les teignes par Plato, Mario Truffi, Bruno Bloch, Ravaut, et dans mon propre laboratoire, car, à mon avis, elles ont dans le sujet une importance capitale.

En quatre matras séparés, contenant un même bouillon stérile, nous pratiquons une culture des quatre types principaux reconnus par la clinique: Achorion banal (culture glabre), Trichophyton banal (culture cartonneuse poudreuse), Microsporum vivace (culture duveteuse devenue opaque) et Microide (culture duveteuse devenue plâtreuse).

Après deux mois, nous stérilisons ces cultures et nous les filtrons sur papier. L'expérience montre que ces bouillons contiennent les poisons solubles sécrétés par ces organismes divers. Nous aurons done une favine, une trichophytine, une microsporine, etc...

Prenons maintenant un malade ayant présenté une dermatophytie suppurée, et, par exemple, un palefrenier ayant présenté (fût-ce deux ans-auparavant) un kérion dù à la teigne du cheval (Trichophyton gypseum, culture plàtreuse). Injectons en un point 
quelconque de sa peau une goutte de l'un quelconque de ces bouillons, il réagira de la même façon. Après trois jours, il présentera un médaillon ovale de rougeur desquamante, de trois centimètres sur deux, qui durera plusieurs semaines, et cette lésion, jamais un autre sujet ne la présentera s'il n'a pas été atteint précédemment d'une dermatophytie suppurée. Et cette réaction ne se produira pas davantage sur le sujet de la première expérience, si au lieu d'un bouillon fait avec un de nos dermatophytes, on se sert d'un bouillon où l'on a cultivé par exemple le parasite de la sporotrichose (Sporotrichum beurmanni).

Ainsi donc, une teigne suppurée quelconque sensibilise également la peau d'un sujet aux toxines de tous les autres dermatophytes, aussi bien qu'à la toxine de eelui qui avait causé la première lésion. De cette expérience capitale une conclusion précise se dégage : quel que soit le type objectif que prennent en culture tous les dermatophytes qui font les teignes, leurs poisons solubles sont si semblables que dans les expériences physiologiques on peut se servir de l'un comme de l'autre indifféremment, et prendre l'un pour l'autre. Ils sont comme interchangeables. A tout esprit tant soit peu familier avec ces questions, cette expérience et ses résultats paraîtront, dans le sujet, de toute importance dogmatique.

Parlant ailleurs de ce fait, j'ai évoqué une comparaison qui s'imposera surtout aux botanistes : Le frêne, le troëne, le lilas, l'olivier ont été rangés par les botanistes dans la même famille (Oléacées) en raison de la morphologie de leur fleur, mais ce qui prouve le mieux que les membres de cette famille ont bien entre eux les affinités que nos classifications leur supposent, c'est que la cantharide, quand elle ne rencontre pas de frêne, se nourrira de lilas ou de troëne, à l'exception de tous autres arbres.

Ce que nous montre l'expérience précédente, c'est la parenté foncière la plus étroite entre les divers parasites de toutes les teignes et leur analogie fondamentale, quelle que soit d'ailleurs l'apparence glabre ou duveteuse de leur culture, et la forme plus ou moins différenciée de leurs organes de reproduction. Voilà un fait vérifiable, dont on peut partir, parce qu'il est expérimental, et d'un plan supérieur à toute discussion morphologique d'école.

Done si nous admettons des groupes naturels parmi les différents dermatophytes, au moins ne devrons-nous pas perdre de vue qu'à travers toutes leurs différences apparentes les espèces les plus dissemblables appartiennent à des êtres extrêmement proches les uns des autres ; ce sont des frères ou au moins des cousins germains. Ce serait donc une erreur certaine de les disperser aux quatre 
coins d'une classification générale. Et le dermatologiste se rappellera que tous, au début de leur inoculation à la peau glabre, y peuvent déterminer un cercle, très analogue pour chacun, $d$ " " herpès circiné n.

Garactères morphologiques parasitaires. - Envisageons maintenant le côté dermatologique de la question et passons à l'étude microscopique de ces parasites sur le vivant. L'expérience montre d'abord que, dans l'épiderme corné de surface, aucun d'eux ne prend une morphologie différentielle. Tous se présentent à nous sous la forme d'un lacis de filaments droits ou courbes, ce sont toujours les "tiges de bambous" de notre comparaison première.

Mais dans le cheveu ou le poil de l'homme ou des animaux, il n'en est pas de même. Suivant le parasite en cause, sa morphologie se personnalise, et cela d'une façon tellement spécifique que, sur leur seul aspect, Gruby en 1842-45, a différencié quatre sur cinq des types que nous connaissons aujourd'hui, et il les a décrit avec une précision qui ne peut laisser place à aucune équivoque.

Et, non seulement Gruby a reconnu ces types microscopiques comme différents, mais comme spécifiques, ayant vérifié déjà ce qui fut vérifié sur des milliers de sujets après lui, que, sur la même tête malade, tous les cheveux atteints montraient un parasite de même morphologie, de même habitat et de même disposition parasitaire. Cette morphologie différentielle étant vraisemblablement connue de presque tous nos lecteurs, je ne m'attarderai pas à la décrire minutieusement et remplacerai cette description par une figure relevée à la chambre claire.

I. - Voici le cheveu de l'Achorion du favus : mycéliums clairs, rectilignes ou sinueux, gros et minces dans le même cheveu, faits d'articles longs ou courts sur le même trajet filamenteux, et pouvant se diviser par tri et tétratomie (tarses faviques). Le parasite n'occupe jamais la totalité du cheveu et laisse voir sa substance propre (fig. 8).

II. - Voici le cheveu de la trichophytie : parasite exclusivement fait d'articles égaux, de forme sporulaire, disposés en files mycéliennes régulières, occupant la totalité du cheveu et remplaçant sa propre substance (ce qui rend le cheveu cassant et fait la teigne tondante) (fig. 9).

III. - Voici le cheveu de la microsporie : cheveu revêtu d'une écorce de fines « spores », polyédriques par pression réciproque, et revêtant le cheveu d'une tunique continue. A l'intérieur du cheveu, rares filaments peu visibles faits d'articles longs entièrement différents des éléments sporulaires de l'écorce (fig. 10). 
IV. - Voici le cheveu des Trichophyton microüdes: revêtus d'une tunique d'éléments très analogues aux précédents, mais agminés en filaments réguliers, en longues files dissociables (fig. 11).

V. - Enfin, voici le cheveu des Trichophyton mégaspores. Ici, la tunique sporulaire du cheveu est faite de gros éléments doubles ou triples de ceux que montre le cheveu microsporique (fig. 12).

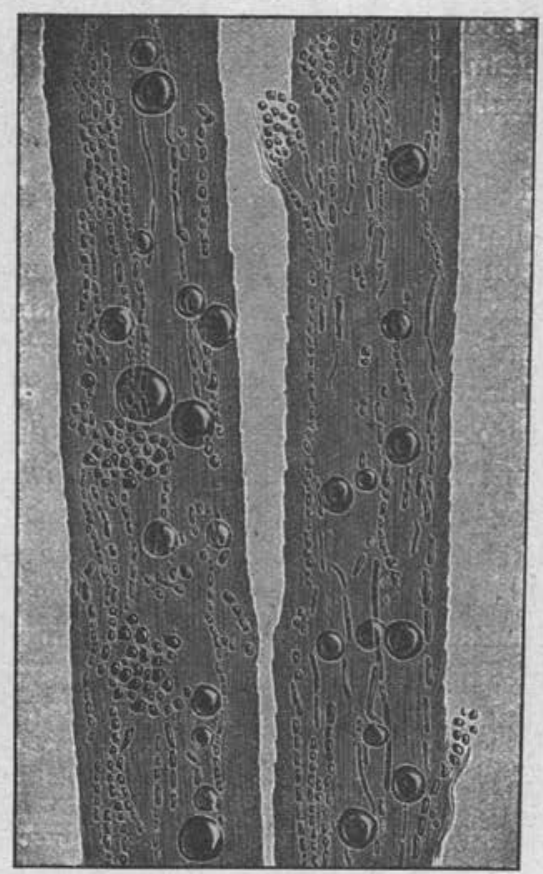

Fig. 8. - Cheveu favique $\times 260$. Préparation du Dr Sabouraud. Dessin de A. Bessin.

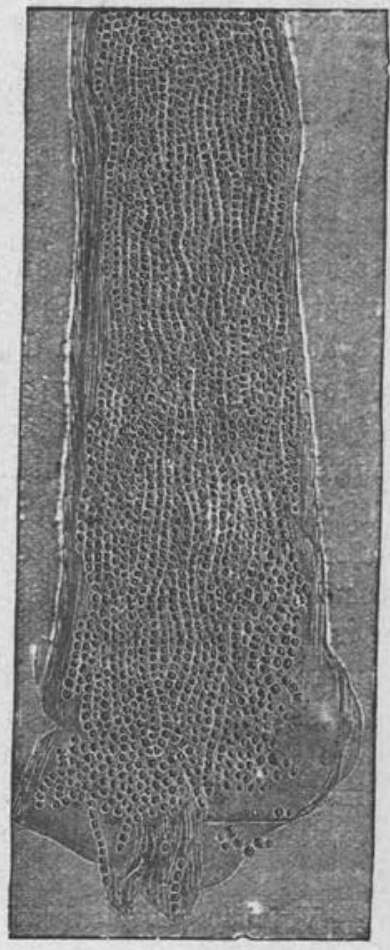

FIc. 9. - Cheveu trichophytique $\times 260$. Préparation du Dr Sabouraud. Dessin de A. Bessin.

Dans un laboratoire consacré à de telles études, l'examen microscopique, pratiqué vingt fois par jour, sera considéré comme si probant que le moindre examen dira à quel type familial appartiennent les cheveux parasités, même si l'observateur n'a pas vu le malade auquel ils appartenaient. Avec un cheveu trichophytique, on n'obtiendra jamais la culture d'un Achorion, ni avec un cheveu microsporique la culture d'un mégasporé. Cela ne se voit jamais.

Sans doute, après cet examen, ne peut-on pas dire la culture 
même que l'on obtiendra, puisqu'il y a plusieurs Microsporum, plusieurs Trichophyton, et que la culture en est différente. Mais on peut dire, à coup sûr, quelle culture ce cheveu ne donnera pas : ce sera toutes celles qui correspondent à une autre disposition du parasite dans le cheveu. Et, non seulement la morphologie du parasite sera constante et par exemple la culture du Trichophyton

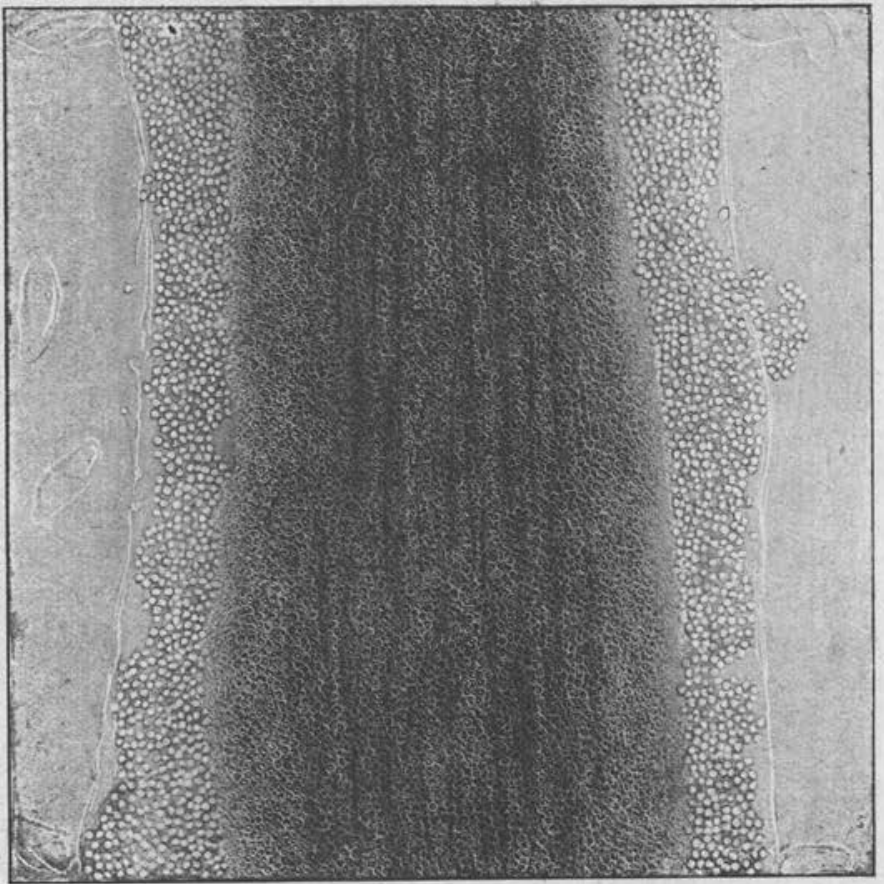

Fıg. 10. - Cheveu microsporique $\times 260$. Préparation du Dr Sabouraud. Dessin de A. Bessin.

crateriforme proviendra toujours d'un parasite endothrix tel que le représente la figure 9. Mais si on inocule cette culture au cobaye, le parasite y reprend exactement la même disposition intra-pilaire, faite des mêmes éléments mêmement disposés.

Il apparait donc au clinicien, et peut-être en apparaîtrait-il de même aux mycologues, s'ils en étaient mieux informés, que cette morphologie si différentielle pour chaque type parasitaire et immuable pour chacun, correspond à quelque chose de familial et peut servir à l'établissement d'une classification ou tout au moins d'une nomenclature rationnelle. C'est en tous cas ce que les dermatolo- 
gistes ont pensé. Pour eux, est appelé Trichophyton endothrix tout parasite ayant les caractères de la figure 9 , etc., etc...

Ici, je prie instamment ceux qui seraient disposés à ne pas croire aveuglément, de bien vouloir renouveler ces expériences. Il n'y a pas besoin d'un service de malades pour cela, des cobayes suffiront à en faire la démonstration. Non seulement, je ne trouverais pas

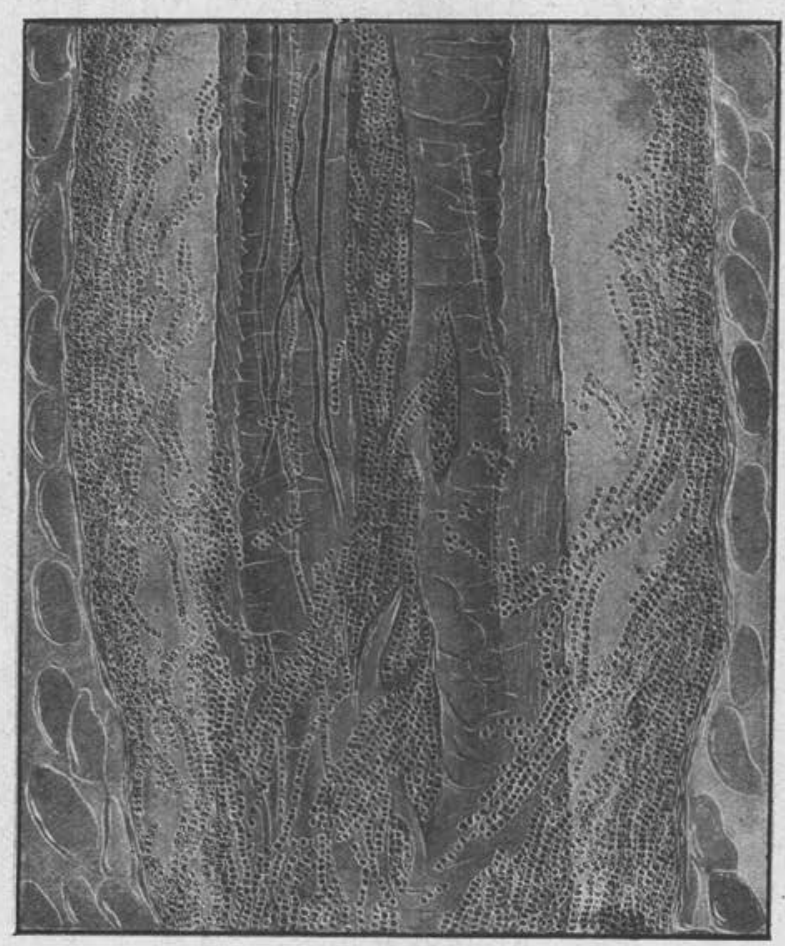

F1G. 11. - Poil envahi et entouré par un microïde $\times 260$. Préparation du Dr Sabouraud. Dessin de A. Bessin.

mauvaise une telle défiance, car il est extrêmement scientifique de ne jamais rien croire sur parole, mais je serais très disposé, au contraire, à traiter comme sans valeur toute série d'expériences qui n'aurait pas commencé par celles-là. Le sujet est assez obscur pour qu'on n'éteigne pas, de parti pris, une des lumières qui s'offrent à nous pour le mieux connaître.

Ainsi donc, les dermatologistes, ayant observé la morphologie parasitaire constante des divers dermatophytes dans le cheveu, ont 
pris pour base de leur nomenclature la forme spéeiale et spécifique du parasite dans le cheveu. Mais on conçoit assez bien que les

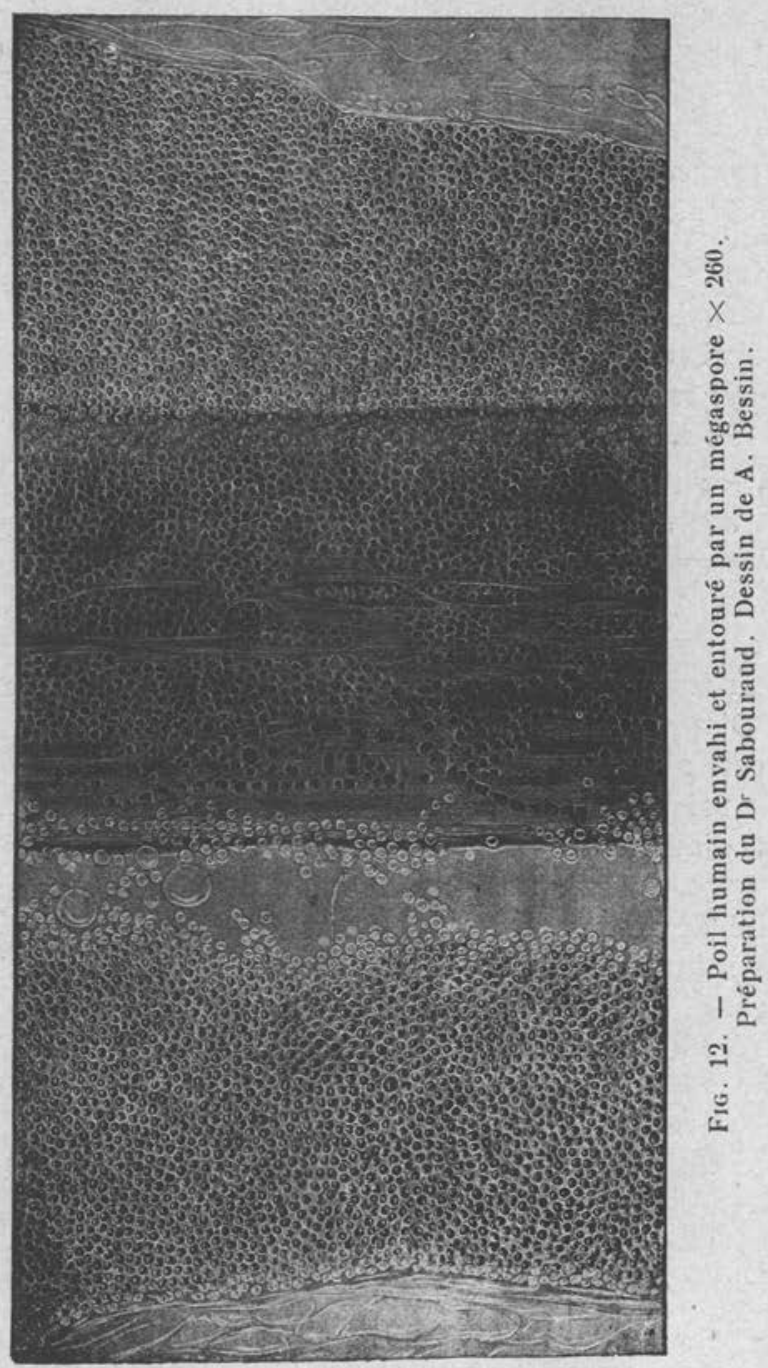

mycologues, à qui les techniques d'inoculationr ne sont pas familières, n'aient pas compris la valeur de cette morphologie spécifique, ni pris la peine de la vérifier sur le cobaye. Ils ne la connaissent que par des textes ou des figures dont ils n'ont mesuré ni l'exacti-

ANnales de PARAsitologie, T. VII, $\mathrm{N}^{\circ} 3 .-1^{\text {er }}$ mai 1929.

16. 
tude ni la précision. Alors, ils sont enclins naturellement à traiter nos expériences avec le même dédain, que nous leurs classifications qui n'en tiennent pas compte. Ef c'est précisément contre ces fâcheuses tendances et ces techniques défectueuses que je me suis élevé, parfois avec un peu de vivacité.

Le mieux à faire pour chacun de nous est de chercher très simplement d'où proviennent nos dissentiments, pour chercher à les effacer. C'est ce que nous allons faire maintenant et nous allons voir que ce ne serait peut-être pas très difficile.

Discussion. - Nous l'avons dit plus haut ; quoique indéfiniment variable dans le détail, la morphologie des appareils de reproduction des dermatophytes est vraiment simple dans son ensemble. Tous réunis ne présentent guère que quatre appareils reproducteurs plus ou moins fréquents :

$1^{\circ}$ Chez les uns, ce sont de simples condensations protoplasmiques dans des cellules mycéliennes distendues, et de paroi renforcée. C'est ce que les mycologues nous disent d'appeler des arthrospores.

$2^{\circ}$ Chez d'autres, de petites cellules de forme globuleuse ou ovoïde, sessiles ou pédiculées, appendues au long d'un filament mycélien. D'après les mycologues, on doit les appeler des aleuries. Souvent, ces aleuries sont agminées en grappes analogues à des grappes de raisin.

$3^{\circ}$ Chez d'autres ou chez les mêmes, on trouve des fuseaux, petits ou gros, ou moyens, non septés, uni ou pluriseptés, mais dont l'unité de forme et d'origine est évidente, qu'ils soient latéraux ou terminaux, peu ou très nombreux, petits ou gros.

$4^{\circ}$ Et, enfin, chez quelques espèces rares, présentant les divers organes précédents, on trouve des vrilles ou spirales, latérales aux filaments mycéliens, en tout semblables aux vrilles de la vigne.

Et c'est tout.

Eh bien, réunissons ces quatre organes en quatre cases côte à côte et construisons av̉ec elles une table à double entrée semblable à la table de Pythagore. Si nous la lisons verticalement, nous aurons à très peu près les familles désignées par les mycologues. Et, si nous la lisons horizontalement, nous aurons les familles indiquées par les dermatologistes, suivant les caractères des parasites dans le cheveu ou le poil. Il me semble que ce tableau, tout schématique qu'il puisse paraître, n'en est pas moins saisissant. Il montre en somme que toutes les catégories cliniques admises en dermatologie montrent toutes, ou à peu près toutes, les formes de reproduction attribuées aux divers dermatophytes. 
Prenons les parasites qui font le favus (1). L'Achorion schönleinii, le prototype de cette famille clinique ne montre en culture que des arthrospores et de très rares aleuries, mais l'Achorion quinckeanum montre de petits fuseaux, plus gros chez l'Achorion gallinæ, innombrables et énormes chez l'Achorion gypseum.

Prenons les Trichophytons endothrix. L'un d'eux, le $T$. violaceum à culture glabre ne montre que des arthrospores (Les teignes, fig. 342), mais les autres, des aleuries innombrables, en thyrses et en grappes. Et, sur plusieurs, on retrouve même des chlamydospores terminales ovoïdes occupant la place des fuseaux (Les teignes, fig. 276-296).

Prenons les Microsporum d'origine humaine, à culture de développement pauvre, nous en voyons de glabres : Microsporum ferrugineum d'Ota faite d'arthrospores ; chez le $M$. audouini, des aleuries rares et caduques, mais des fuseaux petits et nombreux (Les teignes, fig. 361-372). Parmi les Microsporum animaux à culture floride, nous n'avons plus d'espèces glabres, mais il suffira d'en fouiller de vieilles cultures pour y retrouver la reproduction arthrosporée. On y trouve des aleuries rares et des fuseaux géants, nombreux à couvrir les préparations (Les teignes, fig. 373-392).

Quant aux mégaspores glabres (faviformes), ils ne montrent que des arthrospores, identiques d'ailleurs à celles qu'ils montrent dans la vie parasitaire (Les teignes, fig. 343-347). Mais les espèces velues comme le $T$. equinum montrent des aleuries et le $T$. rosaceum, avec les aleuries, des rudiments de petits fuseaux (Les teignes, fig. 335339 ).

Restent les microïdes chez qui tous les organes de reproduction existent : arthrospores dans les vieilles cultures, aleuries partout et en grappes énormes, fuseaux latéraux et terminaux, servant quelquefois de pédicules aux grappes d'aleuries, et enfin les 'spirales spéciales à deux espèces de ce groupe (Les teignes, fig. 297-327).

Résumé et conclusions. - Et maintenant, quelle conclusion tirer de cette révision rapide, sinon celle-là même que les expériences physiologiques ont démontré, à savoir que tous ces parasites sont aussi proches que possible les uns des autres. Dans chaque groupe déterminé par l'examen du cheveu ou du poil, on trouve des cultures glabres (arthrospores), poudreuses (aleuries), ou duveteuses (fuseaux). Et ces différences n'entament pas leur proche parenté

(1) On peut suivre sur les figures correspondantes de mon ouvrage sur Les teignes, l'exactitude de tous ces faits, et comme il a été publié en 1910, avant toutes les récentes discussions mycologiques sur le sujet, leur réalité n'en sera que mieux établie. Voyez pour le texte précédent : fig. 393-415. 
R. SABOURAUD

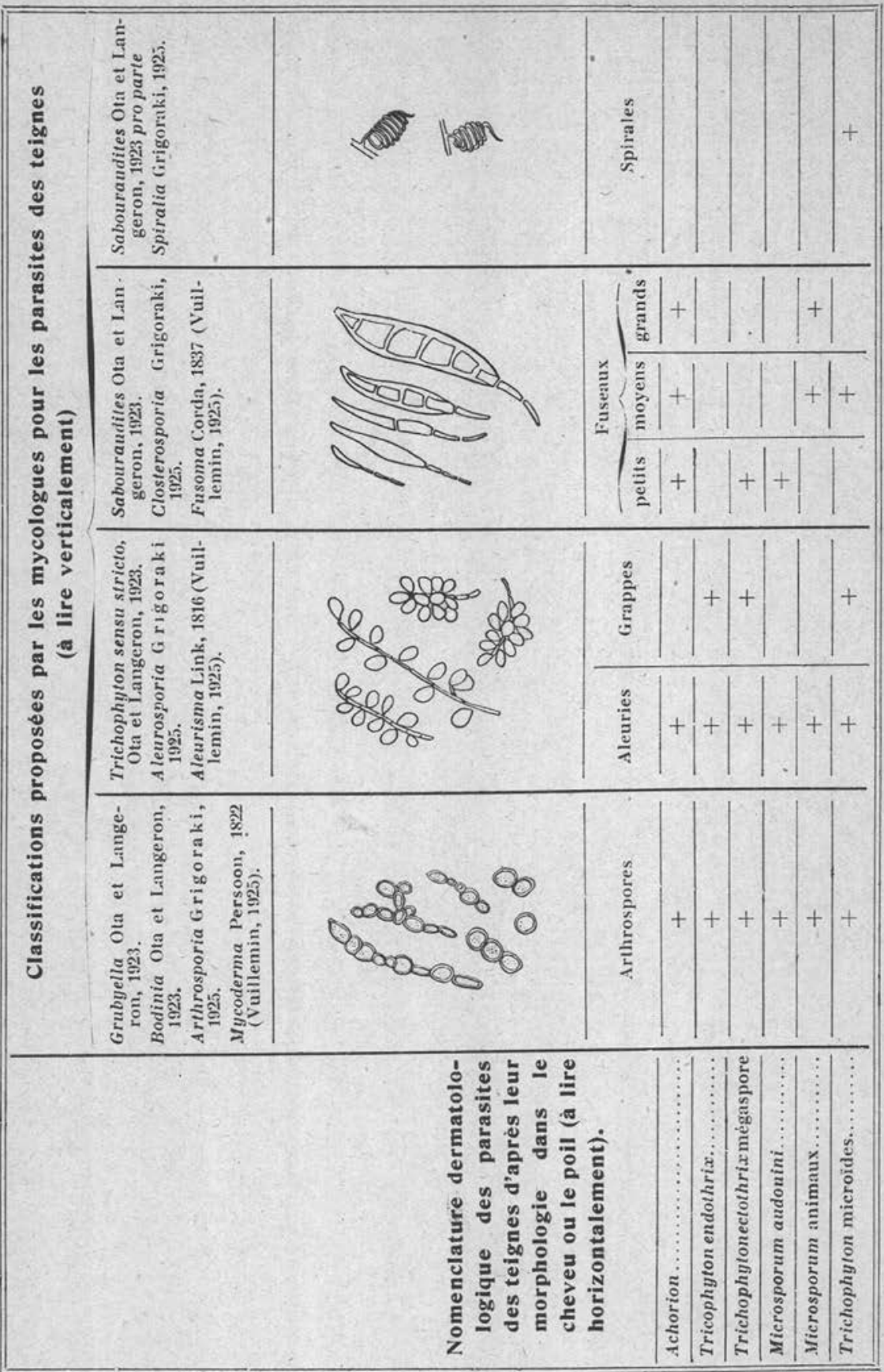


que la mème allergie à toutes leurs toxines démontre au physiologiste. Et ces cadres correspondent aussi exactement à ce que la clinique nous montre. Par son aspect, ses caractères objectifs et microscopiques, sa contagiosité si peu marquée et sa permanence, le favus causé par les Achorion glabres ou duveteux s'éloigne de l'aspect, des mœurs, des caractères objectifs et microscopiques, de l'épidémicité énorme et de la guérison spontanée des microspories à la puberté, quel que soit le Microsporum qui les cause.

Et cela n'est-ce pas une preuve analogue à celle que la cantharide nous donne de la parenté réelle du frêne, du troëne et du lilas ? Au contraire, lisons verticalement ce tableau, nous aurons, par exemple, dans une mème colonne, toutes les cultures glabres ( $1^{\text {re }}$ colonne), mais les unes donneront lieu à un favus, les autres à une tondante, et l'examen microscopique deviendra aussi discordant que le tableau clinique lui-même. Cela n'est-il pas illogique au suprême point, quand on connait la constance et la spécificité des caractères morphologiques de ces parasites dans le cheveu ?

Voilà pourquoi je lis ce tableau dans le sens horizontal, parce que, dans ce sens, tous les recoupements que la physiologie, la clinique et le microscope nous fournissent se trouvent exacts.

Est-ce à dire que je vois les classifications des teignes, telles que les ont faites les dermatologistes, immuables et définitives ? Pas le moins du monde.

J'avais placé les microüdes parmi les Trichophyton, est-ce bien leur place ? Ils seraient aussi bien appelés microìdes tout court et placés à còté des Microsporum, animaux dont ils se rapprochent grandement.

On a nommé d'un nom particulier, comme espèces, toutes les variétés qui, en cultures sériées, se sont montrées fixes en leurs caractères. Sont-elles espèces ou variétés ? Il est certain que le Microsporum lanosum et le $M$. felineum ne se distinguent que par la couleur du centre de leur culture. Mais, cette différence persiste depuis quinze ans... Nous ne nous sommes pas cru autorisés à les confondre. Il semble de mème que le Trichophyton asteroïdes et le $T$. radiolatum ne soient que des variétés fixes d'une même espèce...

On me dirait encore que les $T$. niveum ne sont que des gypseum spontanément pléomorphisés sur le vivant, je le croirais volontiers, si quelqu'un pouvait en donner la preuve.

Et puis, il y a les parasites " hors cadre " comme cet Epidermophyton inguinale si spécial, qui n'attaque jamais le poil et dont les cultures ne montrent rien que des régimes de fuseaux obtus, dressés, sans aucune aleurie. Où le placer ? Nous n'en savons rien. Et 
comme il n'est pas inoculable, sa classification demeure en suspens.

Et puis, les Epidermophyton des lésions interdigitales des pieds et des mains, dont sept espèces sont aujourd'hui différenciées par Kauffmann-Wolf, Priestley, Ota, Bang, Sicoli, Mac Carthy, etc... Ce sont là sujets à l'étude, et sur lesquels il ne faut pas conclure précipitamment.

J'admets donc tout à fait que cette question intéressante puisse sans cesse bénéficier des travaux d'autrui. J'ai voulu montrer seulement que la direction exclusivement morphologique de certains travaux de mycologie pure, au lieu d'améliorer nos connaissances sur le sujet risquait d'ajouter aux obscurités qu’il garde. 